\title{
Grob-type fragmentation of a carvone derived $\beta$-hydroxymesylate: application to the synthesis of chiral lavandulol derivatives
}

\author{
Goverdhan Mehta, ${ }^{a}$ Swastik Karmakar ${ }^{\mathrm{b}}$ and Shital K. Chattopadhyay,* \\ ${ }^{a}$ Department of Organic Chemistry, Indian Institute of Science, Bangalore 560 012, India \\ ${ }^{\mathrm{b}}$ Department of Chemistry, University of Kalyani, Kalyani 741235, West Bengal, India
} Abstract-Grob-type fragmentation of the carvone derived diol-monosulphonate $\mathbf{5}$ has been utilised for the enantioselective synthesis of
various lavandulol derivatives

\section{Introduction}

Although the cyclic template of the monoterpene carvone has been extensively utilised as a chiral-pool material for the asymmetric synthesis of diverse cyclic structures of interest, its application in the synthesis of acyclic chiral molecules is somewhat less documented. ${ }^{1}$ During the course of our studies $^{2}$ towards the construction of taxoids from carvone, we were attracted to the possibility of developing new routes to some acyclic chiral molecules of interest from carvone using suitable ring-opening protocol. Herein, we describe our efforts towards the synthesis of various chiral lavandulol derivatives from carvone. Our strategy relied on the identification of the stereogenic center at C-5 of carvone as identical to that of lavandulol at $\mathrm{C}-2$ and some restructuring plans for the synthesis of various lavandulol derivatives could also be envisioned (Fig. 1).
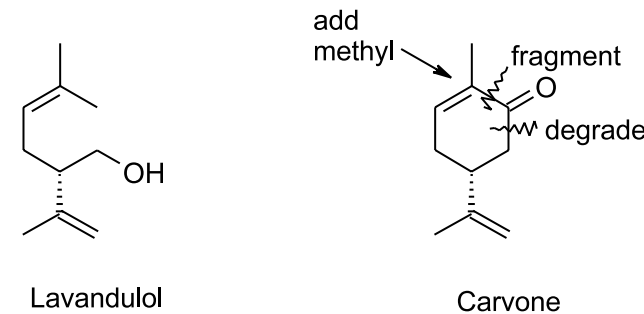

Figure 1. Structural correlation of lavandulol with carvone.

\footnotetext{
Keywords: Fragmentation; Carvone; Lavandulol; Enatioselective.

* Corresponding author. Tel.: +91-33-2582-8750; fax: +91-33-2582-8282; e-mail address: skchatto@yahoo.com
}

\section{Results and discussion}

Our synthetic approach commenced from the cheaper, more abundant $R(-)$-carvone (1) which was readily elaborated to the hydroxyketone $\mathbf{3}$ via a two step sequence involving reductive methylation of the derived epoxyketone $\mathbf{2}$ as described previously. ${ }^{3}$ In line with our earlier observation, ${ }^{2}$ addition of methylmagnesium iodide to $\mathbf{3}$ proceeded with high diastereoselectivity and the diol 4 was obtained in good yield after removal of the minor isomer $(5 \%)$. Selective mesylation of the secondary hydroxy group in the diol $\mathbf{4}$ could easily be accomplished using conventional conditions to yield the hydroxymesylate $\mathbf{5}$ in good yield (Scheme 1).

The Grob-type fragmentation of 1,3-diolmonosulphonates has evolved ${ }^{4}$ into an outstanding piece and its utility in the construction of functionalised alkenes with defined regioand/or stereospecificity has rendered it a valuable tool in organic synthesis. Recently an elegant example of this reaction, for the synthesis of some musk compounds, has been reported. ${ }^{5}$ We considered application of this type of fragmentation of the hydroxymesylate $\mathbf{5}$ to unravel the framework of the lavandulyl system. Pleasingly, the hydroxymesylate $\mathbf{5}$ underwent smooth conversion in refluxing tetrahydrofuran in the presence of sodium hydride to the unsaturated ketone $\mathbf{6}$ which was obtained as a pleasant smelling colourless liquid.

The ketone 6 contains most of the structural features of the important irregular mono-terpene alcohol lavandulol, therefore, a further degradation to lavandulol ${ }^{6}$ was then considered. Although several possibilities exist for this seemingly simple transformation, we argued that a successful Bayer-Villiger oxidation ${ }^{7}$ would convert the ketone 6 

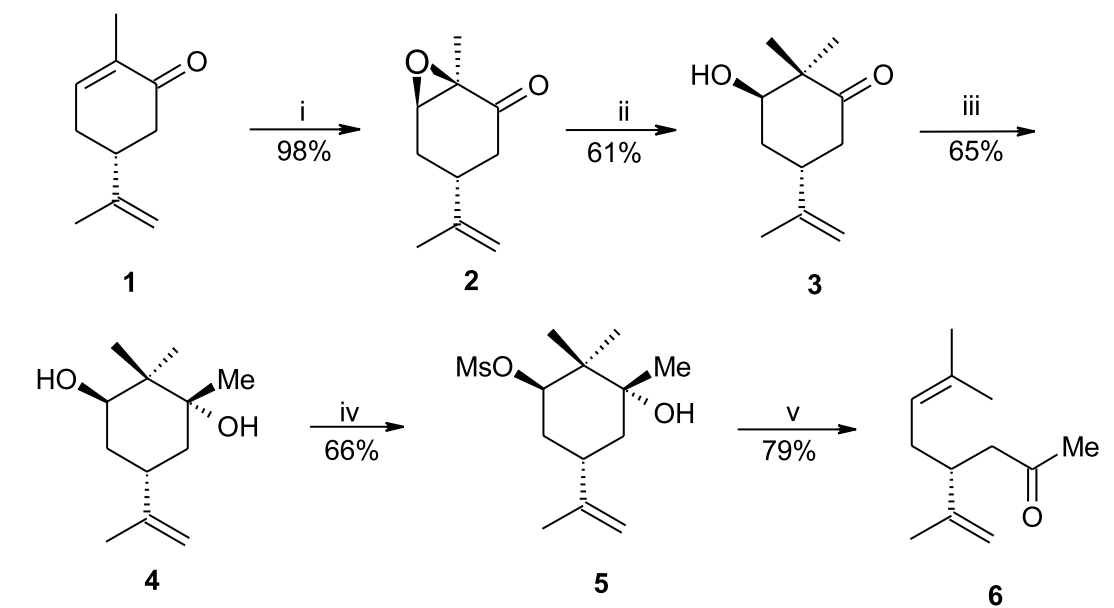

Scheme 1. Reagents and conditions: (i) $\mathrm{H}_{2} \mathrm{O}_{2}, \mathrm{NaOH}, 0{ }^{\circ} \mathrm{C}, 4 \mathrm{~h}, 98 \%$; (ii) $\mathrm{Li}, \mathrm{NH}_{3}, \mathrm{MeI},-33{ }^{\circ} \mathrm{C}$ to room temperature, $3 \mathrm{~h}, 61 \%$; (iii) $\mathrm{MeMgI}, 0{ }^{\circ} \mathrm{C}$ to room temperature, $12 \mathrm{~h}, 65 \%$; (iv) $\mathrm{MsCl}$, pyridine, $0{ }^{\circ} \mathrm{C}$ to room temperature, $8 \mathrm{~h}, 66 \%$; (v) $\mathrm{NaH}, \mathrm{THF}$, reflux, $8 \mathrm{~h}, 79 \%$.

into lavandulol acetate. We were aware of the possibility of competing oxidation of the two somewhat activated double bonds present in the molecule, but ample literature precedence in analogous cyclic substrates prompted us to try some of these fruitful conditions. In all of the experiments with meta-chloroperbenzoic acid (using sodium bicarbonate, para-toluenesulphonic acid, trifluoroacetic acid, etc. as additives) or hydrogen peroxide (in conjunction with acetic or trifluoroacetic acid) epoxidation of the $\mathrm{C}_{6}-\mathrm{C}_{7}$ double bond was the major phenomenon observed, while from basic conditions $\left(\mathrm{H}_{2} \mathrm{O}_{2} / \mathrm{NaOH}\right)$ the starting material was recovered unchanged, even after prolonged heating. Bis-trimethylsilyl peroxide has been reported $^{8}$ to carry-out Bayer-Villiger oxidation of unsaturated ketones but moderate yields have been recorded in most cases. This reagent in conjunction with $\mathrm{SnCl}_{4}$ did indeed afforded lavandulol acetate, but in poor yield (Scheme 2). On the other-hand, the saturated ketone 8 , obtained by hydrogenation of $\mathbf{6}$, underwent smooth BayerVilliger oxidation with sodium percarbonate ${ }^{9}$ in the presence of trifluoroacetic anhydride ${ }^{10}$ to give the acetate 9 in good yield. Hydrolysis of the latter with aqueous potassium carbonate afforded tetrahydrolavandulol in high

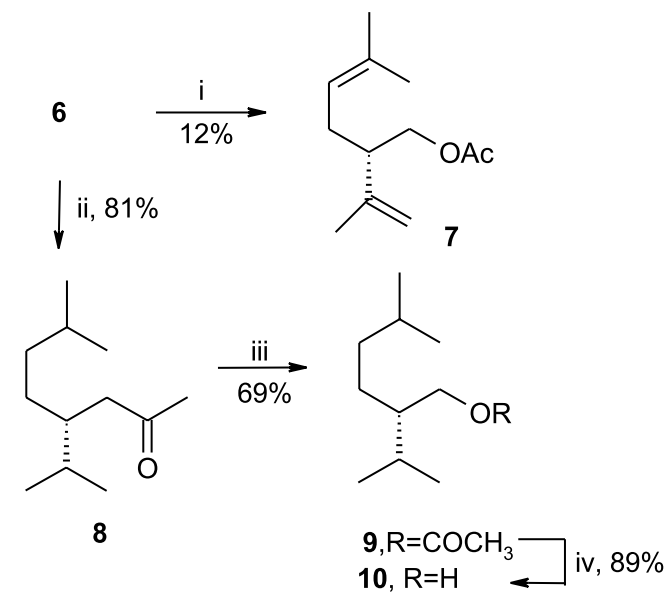

Scheme 2. Reagents and conditions: (i) $\mathrm{TMS}_{2} \mathrm{O}_{2}, \mathrm{SnCl}_{4}, \mathrm{CH}_{2} \mathrm{Cl}_{2}, 24 \mathrm{~h}$, $12 \%$; (ii) $\mathrm{H}_{2}, \mathrm{Pd}-\mathrm{C}$, EtOH, room temperature, 4 h, $81 \%$ (iii) SPC, TFAA, room temperature, $16 \mathrm{~h}, 69 \%$; (iv) potassium carbonate, methanol, room temperature, $2 \mathrm{~h}, 89 \%$. yield. Tetrahydrolavandulol has been utilised as a key intermediate in the synthesis ${ }^{11}$ of tetradesoxybacterioruberin and recently, some interesting biotransformation of the former has also been reported. ${ }^{12}$

We also considered the Beckmann-type rearrangement of the oxime of the ketone $\mathbf{6}$ as an additional possibility. Thus, the ketone 6 was converted into its oxime 11 ( $\sim 7: 1$ mixture of E- and Z-) by treatment with hydroxylamine hydrochloride under conventional conditions. ${ }^{13}$ Treatment $^{14}$ of this mixture with para-toluenesulfonyl chloride in pyridine led to smooth formation of the rearranged product $\mathbf{1 2}$ as the only isolable product (Scheme 3).

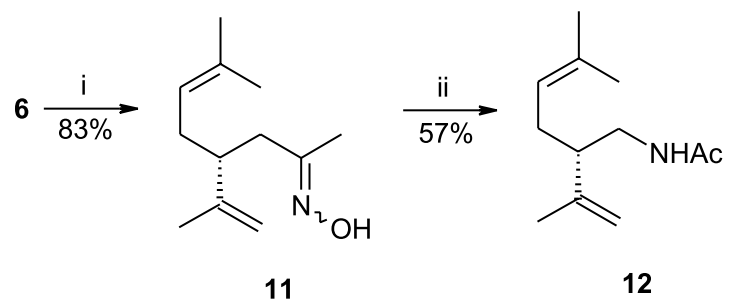

Scheme 3. Reagents and conditions: (i) $\mathrm{NH}_{2} \mathrm{OH} \cdot \mathrm{HCl}$, pyridine, ethanol, rt, $6 \mathrm{~h}, 83 \%$; (ii) $p$-TsCl, pyridine, benzene, rt, $12 \mathrm{~h}, 57 \%$.

\section{Conclusion}

In short, we have demonstrated that various chiral lavandulol derivatives could be prepared from carvone through a fragmentation-based approach. Some of the compounds reported here have the potential to find applications in perfumery.

\section{Experimental}

\subsection{General details}

Optical rotations were recorded in spectroscopic grade chloroform or dichloromethane on a Jasco DIP370 polarimeter, $[\alpha]_{\mathrm{D}}$ values are recorded in units of $10^{-1} \mathrm{deg} \mathrm{cm}^{2} \mathrm{~g}^{-1}$. Infrared spectra were obtained using a Perkin-Elmer 1600 series spectrometer as liquid films or 
dilute solutions in spectroscopic grade dichloromethane. Proton NMR spectra were recorded on a Bruker DRX-300 spectrometer as dilute solutions in deuterochloroform. The chemical shifts are quoted in parts per million ( $\mathrm{ppm}$ ) relative to tetramethylsilane as the internal standard and the multiplicity of each signal is designated by the following abbreviations: s, singlet; d, doublet; $t$, triplet; q, quartet; $m$, multiplet. All coupling constants are quoted in Hertz. Carbon-13 NMR spectra were recorded on Bruker DPX-300 spectrometer as dilute solutions in deuterochloroform. Chemical shifts are recorded relative to internal chloroform ( $\delta 77.2$ ) or TMS as standard on a broad band decoupled mode, and the multiplicities determined using a DEPT sequence. Mass spectra were recorded on a JEOL-JMS 600 instrument and elemental analyses were obtained on a Perkin-Elmer 204b elemental analyzer.

4.1.1. $(1 R, 3 R, 5 R)$-5-isopropenyl-1, 2, 2-trimethylcyclohexane-1,3-diol (4). A solution of methylmagnesium iodide ( $2 \mathrm{M}$ in $\mathrm{THF}, 10 \mathrm{ml}, 20 \mathrm{mmol}$ ) was added dropwise over $20 \mathrm{~min}$ to a stirred solution of the hydroxyketone $3^{3}$ (1.64 g, $9.11 \mathrm{mmol})$ in dry $\mathrm{THF}$ under nitrogen at $0{ }^{\circ} \mathrm{C}$ and the resulting yellowish solution was allowed to come to room temperature over $20 \mathrm{~h}$. It was quenched with saturated aqueous $\mathrm{NH}_{4} \mathrm{Cl}$ solution $(10 \mathrm{ml})$ and then extracted with ether $(2 \times 50 \mathrm{ml})$. The combined organic extract was washed successively with water $(2 \times 50 \mathrm{ml})$, brine $(20 \mathrm{ml})$ and then dried $\left(\mathrm{Na}_{2} \mathrm{SO}_{4}\right)$. It was filtered and the filtrate was concentrated to leave the crude product as a pale yellow oil which on chromatography $\left(\mathrm{SiO}_{2}\right)$ (petroleum ether/ethyl acetate, 7:1) afforded the product as a colourless oil $(1.17 \mathrm{~g}$, 65\%). $[\alpha]_{\mathrm{D}}+8.54\left(c, 0.82\right.$ in $\left.\mathrm{CHCl}_{3}\right) . \nu_{\max }$ (neat) 3340, 2920, 1665, 1080 and $885 \mathrm{~cm}^{-1} .{ }^{1} \mathrm{H}$ NMR $(300 \mathrm{MHz}$, $\left.\mathrm{CDCl}_{3}\right): \delta 4.77(1 \mathrm{H}, \mathrm{s}), 4.70(1 \mathrm{H}, \mathrm{s}), 3.62(1 \mathrm{H}, \mathrm{t}$, $J=9.1 \mathrm{~Hz}), 3.12(1 \mathrm{H}, \mathrm{bs}), 2.98-2.64(1 \mathrm{H}, \mathrm{m}), 1.72(3 \mathrm{H}$, s), $1.69-1.57(4 \mathrm{H}, \mathrm{m}), 1.13(3 \mathrm{H}, \mathrm{s}), 1.10(3 \mathrm{H}, \mathrm{s}), 0.88(3 \mathrm{H}$, s). ${ }^{13} \mathrm{C} \mathrm{NMR}\left(75 \mathrm{MHz}, \mathrm{CDCl}_{3}\right): \delta 149.3(\mathrm{~s}), 108.8(\mathrm{t}), 78.6$ (d), $76.0(\mathrm{~s}), 40.7(\mathrm{t}), 39.4(\mathrm{~s}), 33.9$ (t), 33.8 (d), $25.3(\mathrm{q})$, 24.0 (q), 21.0 (q), 20.6 (q). Elemental analyses: C, 72.49\%; $\mathrm{H}, 11.04 \% ; \mathrm{C}_{12} \mathrm{H}_{22} \mathrm{O}_{2}$ requires $\mathrm{C}, 72.68 \% ; \mathrm{H}, 11.18 \% . \mathrm{m} / \mathrm{z}$ (EI, $70 \mathrm{eV}) 180\left(\mathrm{M}^{+}-\mathrm{H}_{2} \mathrm{O}\right), 145$.

4.1.2. (1R,3R,5R)-3-hydroxy-5-isopropenyl-2,2,3-trimethylcyclohexyl methanesulfonate (5). Methanesulfonyl chloride $(0.85 \mathrm{ml}, 11 \mathrm{mmol})$ was added in one portion to a stirred solution of the diol $4(375 \mathrm{mg}, 1.89 \mathrm{mmol})$ in dry pyridine $(10 \mathrm{ml})$ at $0{ }^{\circ} \mathrm{C}$ under nitrogen. It was allowed to come to room temperature and stirring continued for $14 \mathrm{~h}$. The brownish mixture was diluted with water $(50 \mathrm{ml})$ and then extracted with ether $(3 \times 50 \mathrm{ml})$. The combined ether extract was washed successively with water $(3 \times 25 \mathrm{ml})$ and saturated aqueous copper sulfate solution $(2 \times 25 \mathrm{ml})$. It was then dried $\left(\mathrm{Na}_{2} \mathrm{SO}_{4}\right)$, filtered and the filtrate was concentrated to leave the crude product as a brownish oil which on chromatography $\left(\mathrm{SiO}_{2}\right)$ (petroleum ether/ethyl acetate, 6:1) afforded the product as a colourless oil $(343 \mathrm{mg}, 66 \%)$. $[\alpha]_{\mathrm{D}}$ $-19.58\left(c, 2.72\right.$ in $\left.\mathrm{CHCl}_{3}\right) . \nu_{\max }$ (neat) 3560, 1660, 1330, 1165 and $880 \mathrm{~cm}^{-1} .{ }^{1} \mathrm{H}$ NMR $\left(300 \mathrm{MHz}, \mathrm{CDCl}_{3}\right): \delta 4.89$ $(1 \mathrm{H}, \mathrm{s}), 4.64(1 \mathrm{H}, \mathrm{s}), 3.26-3.06(1 \mathrm{H}, \mathrm{m}), 3.03(3 \mathrm{H}, \mathrm{s}), 2.54$ $(1 \mathrm{H}, \mathrm{tt}, J=8.7,4.2 \mathrm{~Hz}), 2.11-2.02(1 \mathrm{H}, \mathrm{m}), 1.82-1.77(2 \mathrm{H}$, m), $1.73(3 \mathrm{H}, \mathrm{s}), 1.63-1.48(1 \mathrm{H}, \mathrm{m}), 1.15(3 \mathrm{H}, \mathrm{s}), 1.12(3 \mathrm{H}$, s), $0.99(3 \mathrm{H}, \mathrm{s}) .{ }^{13} \mathrm{C}$ NMR $\left(75 \mathrm{MHz}, \mathrm{CDCl}_{3}\right): \delta 147.8(\mathrm{~s})$, $109.6(\mathrm{t}), 90.3(\mathrm{~d}), 74.4(\mathrm{~s}), 40.5(\mathrm{t}), 40.4(\mathrm{~s}), 38.9(\mathrm{~d}), 33.7$ (q), $32.2(\mathrm{t}), 24.7(\mathrm{q}), 24.3(\mathrm{q}), 21.0(\mathrm{q}), 20.5(\mathrm{q})$. Elemental analyses: C, 56.27\%; $\mathrm{H}, 8.44 \%$;, $11.31 \% ; \mathrm{C}_{13} \mathrm{H}_{24} \mathrm{SO}_{4}$ requires $\mathrm{C}, 56.49 \% ; \mathrm{H}, 8.75 \% ; \mathrm{S}, 11.60 \%$.

4.1.3. (4R)-4-isopropenyl-7-methyl-6-octen-2-one (6). Sodium hydride (130 $\mathrm{mg}$, excess) was added in one portion to a solution of the hydroxymesylate $\mathbf{5}(114 \mathrm{mg}, 0.41 \mathrm{mmol})$ in dry tetrahydrofuran $(8 \mathrm{ml})$ under nitrogen atmosphere and the resulting mixture was heated to reflux for $8 \mathrm{~h}$. It was then cooled in an ice-bath and quenched by slow addition of methanol $(1 \mathrm{ml})$ followed by saturated aqueous ammonium chloride $(5 \mathrm{ml})$. It was then extracted with ether $(2 \times 25 \mathrm{ml})$ and the combined ether extract was washed with water $(2 \times 20 \mathrm{ml})$, brine $(1 \times 10 \mathrm{ml})$ and then dried $\left(\mathrm{Na}_{2} \mathrm{SO}_{4}\right)$. It was then filtered and the filtrate was concentrated to leave the crude product as a pale yellow oil which on chromatography $\left(\mathrm{SiO}_{2}\right)$ (petroleum ether/ethyl acetate, 20:1) afforded the product as a colourless oil $(58 \mathrm{mg}, 79 \%) .[\alpha]_{\mathrm{D}}+12.1(c, 2.1$ in $\left.\mathrm{CHCl}_{3}\right) . \nu_{\max }$ (neat) $2920,1720,1665$ and $1645 \mathrm{~cm}^{-1} .{ }^{1} \mathrm{H}$ NMR $\left(300 \mathrm{MHz}, \mathrm{CDCl}_{3}\right): \delta 5.01(1 \mathrm{H}, \mathrm{t}, J=7.2 \mathrm{~Hz}), 4.73$ $(1 \mathrm{H}, \mathrm{s}), 4.67(1 \mathrm{H}, \mathrm{s}), 2.60(1 \mathrm{H}$, quin., $J=6.9 \mathrm{~Hz}), 2.46-2.44$ $(2 \mathrm{H}, \mathrm{m}), 2.08(3 \mathrm{H}, \mathrm{s}), 2.03(2 \mathrm{H}, \mathrm{t}, J=7.2 \mathrm{~Hz}), 1.66(6 \mathrm{H}, \mathrm{s})$, $1.54(3 \mathrm{H}, \mathrm{s}) .{ }^{13} \mathrm{C}$ NMR $\left(75 \mathrm{MHz}, \mathrm{CDCl}_{3}\right): \delta 208.4(\mathrm{~s}), 147.1$ $(\mathrm{s}), 133.0(\mathrm{~s}), 122.0(\mathrm{~d}), 111.0(\mathrm{t}), 47.5(\mathrm{t}), 42.8(\mathrm{~d}), 30.1(\mathrm{q})$, 32.0 (t), 25.8 (q), 19.8 (q), 17.8 (q). Elemental analyses: C, $79.69 \% ; \mathrm{H}, 10.98 \% ; \mathrm{C}_{12} \mathrm{H}_{20} \mathrm{O}$ requires $\mathrm{C}, 79.95 \% ; \mathrm{H}$, $11.18 \% . \mathrm{m} / \mathrm{z}$ (EI, $70 \mathrm{eV}) 180\left(\mathrm{M}^{+}\right), 165,137$ (100\%).

4.1.4. (2S)-2-isopropenyl-5-methyl-4-hexenyl acetate (7). Stannic chloride ( $130 \mathrm{mg}, 0.5 \mathrm{mmol}$ ) was added dropwise to a stirred solution of the ketone $(90 \mathrm{mg}, 0.5 \mathrm{mmol})$ and bis(trimethylsilyl)peroxide ${ }^{15} \quad(90 \mathrm{mg}, \quad 0.5 \mathrm{mmol})$ in dichloromethane $(5 \mathrm{ml})$ at $0{ }^{\circ} \mathrm{C}$ under nitrogen atmosphere. After stirring for $1 \mathrm{~h}$ at that temperature it was allowed to come to room temperature and stirring continued for $24 \mathrm{~h}$. It was then diluted with dichloromethane $(25 \mathrm{ml})$ and then poured into an aqueous solution of sodium thiosulfate $(10 \mathrm{ml})$. The organic layer was separated and washed successively with water $(2 \times 10 \mathrm{ml})$, saturated sodium bicarbonate solution $(1 \times 10 \mathrm{ml})$, water $(1 \times 10 \mathrm{ml})$ and brine $(1 \times 10 \mathrm{ml})$. It was then dried $\left(\mathrm{Na}_{2} \mathrm{SO}_{4}\right)$, filtered and the filtrate was concentrated to leave the crude product as a brownish oil which on chromatography $\left(\mathrm{SiO}_{2}\right)$ (petroleum ether/ethyl acetate, 12:1) afforded lavandulol acetate as a colourless oil $(11 \mathrm{mg}, 12 \%)$. $[\alpha]_{\mathrm{D}} 6.3\left(c, 1.5 \mathrm{in} \mathrm{CHCl}_{3}\right) . \nu_{\max }$ (neat) 2910, 1708, 1670 and $1645 \mathrm{~cm}^{-1} \cdot{ }^{1} \mathrm{H} \quad \mathrm{NMR}$ $\left(300 \mathrm{MHz}, \mathrm{CDCl}_{3}\right): \delta 5.05(1 \mathrm{H}, \mathrm{t}, J=7.5 \mathrm{~Hz}), 4.82(1 \mathrm{H}, \mathrm{d}$, $J=1.5 \mathrm{~Hz}), 4.73(1 \mathrm{H}, \mathrm{bs}), 4.03(2 \mathrm{H}, \mathrm{d}, J=6.9 \mathrm{~Hz}) 2.39(1 \mathrm{H}$, quin., $J=6.9 \mathrm{~Hz}), 2.17-2.05(2 \mathrm{H}, \mathrm{m}), 2.02(3 \mathrm{H}, \mathrm{s}), 1.69-$ $1.64\left(6 \mathrm{H}\right.$, overlapping singlets), $1.59(3 \mathrm{H}, \mathrm{s}) .{ }^{13} \mathrm{C} \mathrm{NMR}$ (75 MHz, $\mathrm{CDCl}_{3}$ ): $\delta 171.1$ (s), 144.9 (s), 132.9 (s), 121.6 (d), $112.4(\mathrm{t}), 65.8(\mathrm{t}), 46.0(\mathrm{~d}), 28.5(\mathrm{t}), 25.7$ (q), 20.9 (q), 19.9 (q), 17.8 (q). Elemental analyses: C, 73.66\%; H, $10.49 \% ; \mathrm{C}_{12} \mathrm{H}_{20} \mathrm{O}_{2}$ requires $\mathrm{C}, 73.43 \% ; \mathrm{H}, 10.27 \% . \mathrm{m} / \mathrm{z}(\mathrm{EI}$, $70 \mathrm{eV}) 196\left(\mathrm{M}^{+}\right), 153,59(100 \%)$.

4.1.5. (4R)-4-isopropyl-7-methyloctan-2-one (8). A solution of the ketone 6 (72 $\mathrm{mg}, 0.4 \mathrm{mmol})$ in methanol $(5 \mathrm{ml})$ was vigorously stirred under hydrogen atmosphere in the presence of $\mathrm{Pd}-\mathrm{C}(10 \%, 5 \mathrm{mg})$ for $3 \mathrm{~h}$ at room temperature. The heterogeneous mixture was then filtered through celite and the filter cake was washed with ether. The combined organic solution was then concentrated to leave the crude 
product as a colourless liquid, which was purified by chromatography over silica gel using a mixture of petroleum ether and ethyl acetate (20:1) as eluent. The product was obtained as a colourless liquid $(59 \mathrm{mg}, 81 \%)$. $[\alpha]_{\mathrm{D}}+2.1(c$, 1.2 in $\left.\mathrm{CHCl}_{3}\right) . \nu_{\max }$ (neat) $1710 \mathrm{~cm}^{-1} .{ }^{1} \mathrm{H} \mathrm{NMR}(300 \mathrm{MHz}$, $\left.\mathrm{CDCl}_{3}\right): \delta 2.30(1 \mathrm{H}, \mathrm{dd}, J=15.9,5.7 \mathrm{~Hz}), 2.15(1 \mathrm{H}, \mathrm{dd}$, $J=15.9,5.7 \mathrm{~Hz}), 2.03(3 \mathrm{H}, \mathrm{s}), 1.75(1 \mathrm{H}, \mathrm{m}), 1.65(1 \mathrm{H}, \mathrm{m})$, $1.36(1 \mathrm{H}, \mathrm{m}), 1.21(2 \mathrm{H}, \mathrm{m}), 1.12(2 \mathrm{H}, \mathrm{m}), 0.8(12 \mathrm{H}$, overlapping doublets). ${ }^{13} \mathrm{C} \mathrm{NMR}\left(75 \mathrm{MHz}, \mathrm{CDCl}_{3}\right): \delta 209.8$ $(\mathrm{s}), 45.4(\mathrm{~d}), 39.6(\mathrm{t}), 36.5(\mathrm{t}), 30.3(\mathrm{t}), 29.5(\mathrm{q}), 28.9(\mathrm{~d})$, 28.2 (d), 22.6 (q), 22.4 (q), 19.5 (q), 18.4 (q). Elemental analyses: $\mathrm{C}, 77.88 \% ; \mathrm{H}, 12.90 \% ; \mathrm{C}_{12} \mathrm{H}_{24} \mathrm{O}$ requires $\mathrm{C}$, $78.19 \%$; H, 13.12\%. $\mathrm{m} / \mathrm{z}(\mathrm{EI}, 70 \mathrm{eV}) 184\left(\mathrm{M}^{+}\right), 169,141$ (100\%), 126.

4.1.6. (2S)-2-isopropyl-5-methylhexyl acetate (9). Trifluoroacetic anhydride $(0.085 \mathrm{ml})$ was added to a stirred heterogeneous mixture of the ketone $8(35 \mathrm{mg}, 0.2 \mathrm{mmol})$ and sodium percarbonate $(64 \mathrm{mg}, 0.4 \mathrm{mmol})$ in dry dichloromethane $(2 \mathrm{ml})$ at $0{ }^{\circ} \mathrm{C}$ under nitrogen atmosphere. The mixture was allowed to come to room temperature and stirred for $16 \mathrm{~h}$. It was then diluted with dichloromethane $(20 \mathrm{ml})$ and filtered. The filtrate was concentrated in vacuo and the residue was partitioned between ether $(20 \mathrm{ml})$ and water $(20 \mathrm{ml})$. The organic layer was washed successively with saturated sodium bicarbonate solution $(2 \times 10 \mathrm{ml})$, water $(10 \mathrm{ml})$, brine and then dried $\left(\mathrm{Na}_{2} \mathrm{SO}_{4}\right)$. It was filtered and the filtrate was concentrated to leave the crude product as a pale yellow oil which on chromatography $\left(\mathrm{SiO}_{2}\right)$ (petroleum ether/ethyl acetate, 10:1) afforded tetrahydrolavandulol acetate as a colourless oil $(27 \mathrm{mg}, 69 \%) . \nu_{\max }$ (neat) $1708 \mathrm{~cm}^{-1} .{ }^{1} \mathrm{H}$ NMR $\left(300 \mathrm{MHz}, \mathrm{CDCl}_{3}\right): \delta 3.99-$ $3.84(2 \mathrm{H}, \mathrm{m}), 2.03(3 \mathrm{H}, \mathrm{s}), 1.75-1.66(1 \mathrm{H}, \mathrm{m}), 1.46-1.40$ $(2 \mathrm{H}, \mathrm{m}), 1.37-1.30(1 \mathrm{H}, \mathrm{m}), 1.24-1.16(2 \mathrm{H}, \mathrm{m}), 1.12-1.00$ $(2 \mathrm{H}, \mathrm{m}), 0.88-0.82$ (12H, overlapping doublets), 0.76-0.71 $(1 \mathrm{H}, \mathrm{m}) .{ }^{13} \mathrm{C}$ NMR $\left(75 \mathrm{MHz}, \mathrm{CDCl}_{3}\right): \delta 171.3(\mathrm{~s}), 65.5(\mathrm{t})$, $43.2(\mathrm{~d}), 36.7$ (t), 28.3 (d), 25.7 (t), 22.6 (d), 22.8 (q), 22.4 (q), $21.0(\mathrm{q}), 19.8$ (q), 19.3 (q). Elemental analyses: C, $71.70 \% ; \mathrm{H}, 11.89 \% ; \mathrm{C}_{12} \mathrm{H}_{24} \mathrm{O}_{2}$ requires $\mathrm{C}, 71.95 \% ; \mathrm{H}$, $12.07 \%$.

4.1.7. (2S)-2-isopropyl-5-methylhexan-1-ol (10). A solution of the acetate $9(99 \mathrm{mg}, 0.5 \mathrm{mmol})$ in methanol $(5 \mathrm{ml})$ and saturated aqueous potassium carbonate $(2 \mathrm{ml})$ was stirred at room temperature for $2 \mathrm{~h}$. It was then diluted with water $(20 \mathrm{ml})$ and extracted with ether $(2 \times 20 \mathrm{ml})$. The combined ether extract was washed with brine $(1 \times 10 \mathrm{ml})$ and dried $\left(\mathrm{Na}_{2} \mathrm{SO}_{4}\right)$. It was filtered and the filtrate was concentrated to leave the crude product as a pale yellow oil which on chromatography $\left(\mathrm{SiO}_{2}\right)$ (petroleum ether/ethyl acetate, 10:1) afforded tetrahydrolavandulol as a colourless oil $(71 \mathrm{mg}, 89 \%) .[\alpha]_{\mathrm{D}}-9.6\left(c, 1.4\right.$ in $\left.\mathrm{CHCl}_{3}\right) . \nu_{\max }$ (neat) $3320 \mathrm{~cm}^{-1}$. ${ }^{1} \mathrm{H}$ NMR $\left(300 \mathrm{MHz}, \mathrm{CDCl}_{3}\right): \delta 3.96-3.58(2 \mathrm{H}$, $\mathrm{m}), 1.82-1.78(1 \mathrm{H}, \mathrm{m}), 1.53-1.30(5 \mathrm{H}, \mathrm{m}), 0.91-0.87$ (12H, overlapping doublets). ${ }^{13} \mathrm{C} \mathrm{NMR}\left(75 \mathrm{MHz}, \mathrm{CDCl}_{3}\right): \delta$ $64.2(\mathrm{t}), 47.3(\mathrm{~d}), 37.5(\mathrm{t}), 28.8(\mathrm{~d}), 28.3(\mathrm{~d}), 25.8(\mathrm{t}), 23.1$ (q), 22.9 (q), 20.1 (q), 19.6 (q). Elemental analyses: C, $75.69 \% ; \mathrm{H}, 13.82 \% ; \mathrm{C}_{10} \mathrm{H}_{22} \mathrm{O}$ requires $\mathrm{C}, 75.88 \% ; \mathrm{H}$, $14.01 \%$.

4.1.8. (4R)-4-isopropenyl-7-methyl-6-octen-2-one oxime (11). Hydroxylamine hydrochloride $(232 \mathrm{mg}, 3.34 \mathrm{mmol})$ was added in one portion to a stirred solution of the ketone 6
(500 $\mathrm{mg}, 2.78 \mathrm{mmol})$ in pyridine $(1.25 \mathrm{ml})$ and ethanol $(1 \mathrm{ml})$ and the resulting mixture was stirred for $6 \mathrm{~h}$ at ambient temperature. It was then cooled to $0{ }^{\circ} \mathrm{C}$, diluted with water $(5 \mathrm{ml})$ and extracted with ethyl acetate $(2 \times 10 \mathrm{ml})$. The combined organic extract was washed successively with aqueous hydrochloric acid (5\%, $1 \times 10 \mathrm{ml})$, sodium bicarbonate solution $(5 \%, 1 \times 10 \mathrm{ml})$, water $(1 \times 10 \mathrm{ml})$ and then brine $(1 \times 10 \mathrm{ml})$. It was then dried $\left(\mathrm{Na}_{2} \mathrm{SO}_{4}\right)$, filtered and the filtrate was concentrated in vacuo to leave a pale yellow oil which was purified by chromatography $\left(\mathrm{SiO}_{2}\right)$ (petroleum ether/ethyl acetate, $10: 1)$ to leave the product as a colourless oil $(0.451 \mathrm{~g}$, $83 \%) . \nu_{\max }$ (neat) $3200,2890,1625,1430$ and $1360 \mathrm{~cm}^{-1}$. ${ }^{1} \mathrm{H}$ NMR (300 MHz, $\left.\mathrm{CDCl}_{3}\right)$ : data for the mixture ( $\sim: 1$ by GC) (data in [] refer to the possible $s y n$-isomer) $\delta 5.05(1 \mathrm{H}$, br t), $4.75(1 \mathrm{H}, \mathrm{s})[4.72, \mathrm{~s}], 2.43-2.33(2 \mathrm{H}, \mathrm{m}),[2.10-2.07$ $(\mathrm{m})], 1.85(3 \mathrm{H}, \mathrm{s})[1.84, \mathrm{~s}], 1.68(3 \mathrm{H}, \mathrm{s}), 1.65(3 \mathrm{H}, \mathrm{s}), 1.59$ $(3 \mathrm{H}, \mathrm{s})$. Elemental analyses: $\mathrm{C}, 73.62 \% ; \mathrm{H}, 10.89 \%$; $7.46 \% ; \mathrm{C}_{12} \mathrm{H}_{21} \mathrm{NO}$ requires $\mathrm{C}, 73.80 \% ; \mathrm{H}, 10.84 \%, \mathrm{~N}$, $7.17 \%$.

4.1.9. N 1-[(2S)-2-isopropenyl-5-methyl-4-hexenyl]acetamide (12). $p$-Toluenesulfonyl chloride (341 mg, $1.79 \mathrm{mmol}$ ) was added in one portion to a solution of the oxime $11(350 \mathrm{mg}, 1.79 \mathrm{mmol})$ in a mixture of benzene $(1.5 \mathrm{ml})$ and pyridine $(0.4 \mathrm{ml})$.The resulting homogeneous mixture was stirred at room temperature for $12 \mathrm{~h}$ and then diluted with water $(10 \mathrm{ml})$ and ethyl acetate $(20 \mathrm{ml})$. The aqueous phase was extracted with ethyl acetate $(2 \times 10 \mathrm{ml})$ and the combined organic extract was washed successively with aqueous hydrochloric acid solution $(5 \%, 2 \times 10 \mathrm{ml})$, sodium bicarbonate solution $(5 \%, 1 \times 10 \mathrm{ml})$, water $(2 \times 10 \mathrm{ml})$ and brine $(1 \times 10 \mathrm{ml})$. It was then dried $\left(\mathrm{Na}_{2} \mathrm{SO}_{4}\right)$, filtered and the filtrate was concentrated in vacuo to leave the crude product as a brownish oil which was purified by chromatography $\left(\mathrm{SiO}_{2}\right)$ (petroleum ether/ ethyl acetate, 4:1) to give the product as a colourless oil $(202 \mathrm{mg}, 57 \%) .[\alpha]_{\mathrm{D}}-7.6\left(c, 1.4\right.$ in $\left.\mathrm{CHCl}_{3}\right) . \nu_{\max }$ (neat) 3280, 2900, 1635 and $1540 \mathrm{~cm}^{-1}$. ${ }^{1} \mathrm{H}$ NMR $(300 \mathrm{MHz}$, $\left.\mathrm{CDCl}_{3}\right): \delta 5.41(1 \mathrm{H}, \mathrm{bs}), 5.03(1 \mathrm{H}, \mathrm{t}, J=6 \mathrm{~Hz}), 4.87(1 \mathrm{H}, \mathrm{s})$, $4.77(1 \mathrm{H}, \mathrm{s}), 3.42(1 \mathrm{H}, \mathrm{dt}, J=12.6,5.5 \mathrm{~Hz}), 3.02(1 \mathrm{H}$, ddd, $J=13.4,9.5,4.2 \mathrm{~Hz}), 2.24-2.16(2 \mathrm{H}, \mathrm{m}), 2.04(1 \mathrm{H}$, broad $\mathrm{t}$, $J=6.6 \mathrm{~Hz}), 1.95(3 \mathrm{H}, \mathrm{s}), 1.68(3 \mathrm{H}, \mathrm{s}) 1.65(3 \mathrm{H}, \mathrm{s}), 1.58(3 \mathrm{H}$, s). ${ }^{13} \mathrm{C} \mathrm{NMR}\left(75 \mathrm{MHz}, \mathrm{CDCl}_{3}\right): \delta 170.3(\mathrm{~s}), 145.6(\mathrm{~s}), 132.9$ $(\mathrm{s}), 121.5(\mathrm{~d}), 113.0(\mathrm{t}), 47.1(\mathrm{~d}), 40.9(\mathrm{t}), 29.7(\mathrm{t}), 25.6(\mathrm{q})$, 23.2 (q), 18.6 (q), 17.8 (q). Elemental analyses: C, 73.53\%; $\mathrm{H}, 11.09 \% ; \mathrm{N}, 7.42 \% ; \mathrm{C}_{12} \mathrm{H}_{21} \mathrm{NO}$ requires $\mathrm{C}, 73.80 \% ; \mathrm{H}$, $10.84 \%$; N, 7.17\%. m/z (EI, $70 \mathrm{eV}) 196\left(\mathrm{M}^{+}+1\right), 123,69$ $(100 \%)$.

\section{Acknowledgements}

Financial assistance from the CSIR, New Delhi (Grant No. 00/EMR/II-1676) is gratefully acknowledged.

\section{References and notes}

1. (a) Corey, E. J.; Pearce, H. L. J. Am. Chem. Soc. 1979, 101, 5841. (b) Trost, B. M.; Tasker, A. S.; Ruther, G.; Brandes, A. J. Am. Chem. Soc. 1991, 113, 670. (c) Wender, P. A.; Singh, 
S. K. Tetrahedron Lett. 1990, 31, 3857. (d) Kozikowski, A. P.; Wu, J.-P. Synlett 1991, 465. (e) Srikrishna, A.; Reddy, T. J.; Praveen Kumar, P. J. Chem. Soc., Chem. Commun. 1996, 1369. (f) Meulemans, T. M.; Stork, G. A.; Jansen, B. J. M.; de Groot, A. Tetrahedron Lett. 1998, 39, 6565.

2. Mehta, G.; Chattopadhyay, S. K.; Umarya, J. D. Tetrahedron Lett. 1999, 40, 4881.

3. McChesney, J. D.; Thompson, T. N. J. Org. Chem. 1985, 50, 3473.

4. Weyerstahl, P.; Marschall, H. Comprehensive Organic Synthesis; Trost, B. M., Fleming, I., Winterfeldt, E., Eds.; Pergamon: Oxford, 1991; Vol. 6, pp 1042-1070.

5. Fehr, C.; Galindo, J.; Etter, O.; Thommen, W. Angew. Chem., Int. Ed. 2002, 41, 4523 .

6. (a) Schinz, H.; Siedel, C. F. Helv. Chem. Acta 1942, 25, 1572. (b) For recent reports on the synthesis of lavandulol, see: Piva, O. J. Org. Chem. 1995, 60, 7879. (c) Mino, T.; Fukui, S.; Yamashita, M. J. Org. Chem. 1997, 62, 734.
7. For a review, see: Krow, G. R. Org. React. 1993, 43, 3.

8. Gottlich, R.; Yamakoshi, K.; Sasai, H.; Shibasaki, M. Synlett 1997, 971.

9. For a review, see: McKillop, A.; Sanderson, W. R. J. Chem. Soc., Perkin Trans. 1 2000, 471.

10. Kang, H.-J.; Jeon, H.-S. Bull. Korean Chem. Soc. 1996, 17, 5.

11. Johansen, J. E.; Liaaen-Jensen, S. Tetrahedron Lett. 1976, 12, 935.

12. Nankani, H.; Miyazawa, M.; Kameoka, H. J. Nat. Prod. 1997, 60, 287.

13. Wilson, S. R.; Sawicki, R. A.; Huffman, J. C. J. Org. Chem. 1981, 46, 3887.

14. Lo, F. Y.; Strouse, C. E.; Callahan, K. P.; Knobler, C. B.; Hawthorne, M. F. J. Am. Chem. Soc. 1975, 97, 429.

15. $\mathrm{Me}_{3} \mathrm{SiOOSiMe}_{3}$ was prepared according to the literature: Oswald, A. A.; Guertin, D. L. J. Org. Chem. 1963, 28, 651. 